\title{
Maternal near miss and maternal death in the Pretoria Academic Complex, South Africa: A population-based study
}

\author{
P Soma-Pillay, ${ }^{1} \mathrm{MB}$ ChB, Dip (Obstet) SA, MMed (OetG), FCOG, Cert Maternal and Fetal Medicine (SA); \\ R C Pattinson, ${ }^{2}$ MD, FRCOG, FCOG (SA); , L Langa-Mlambo, ${ }^{3}$ MB ChB, FCOG; B S S Nkosi, ${ }^{4}$ BSc, MB ChB, FCOG, MMed; \\ A P Macdonald, ${ }^{5} \mathrm{MB} \mathrm{ChB}, \mathrm{MMed}(\mathrm{Oet} \mathrm{)}), \mathrm{FCOG}$, FRCOG

\begin{abstract}
${ }^{1}$ Department of Obstetrics and Gynaecology, Faculty of Health Sciences, University of Pretoria, South Africa, and Steve Biko Academic Hospital, Pretoria

${ }^{2}$ South African Medical Research Council Maternal and Infant Health Care Strategies Unit, Department of Obstetrics and Gynaecology, Faculty of Health Sciences, University of Pretoria, South Africa

${ }^{3}$ Maternal and Fetal Medicine Unit, Department of Obstetrics and Gynaecology, Faculty of Health Sciences, University of Pretoria, South Africa

${ }^{4}$ Department of Obstetrics and Gynaecology, Mamelodi Hospital, Pretoria, South Africa

${ }^{5}$ District Clinical Specialist and Department of Obstetrics and Gynaecology, Faculty of Health Sciences, University of Pretoria, South Africa
\end{abstract}

Corresponding author: P Soma-Pillay (priya.somapillay@up.ac.za)

Background. In order to reduce maternal mortality in South Africa (SA), it is important to understand the process of obstetric care, identify weaknesses within the system, and implement interventions for improving care.

Objective. To determine the spectrum of maternal morbidity and mortality in the Pretoria Academic Complex (PAC), SA.

Methods. A descriptive population-based study that included all women delivering in the PAC. The World Health Organization definition, criteria and indicators of near miss and maternal death were used to identify women with severe complications in pregnancy.

Results. Between 1 August 2013 and 31 July 2014, there were 26614 deliveries in the PAC. The institutional maternal mortality ratio was 71.4/100 000 live births. The HIV infection rate was $19.9 \%$, and $2.7 \%$ of women had unknown HIV status. Of the women, 1120 (4.2\%) developed potentially life-threatening conditions and $136(0.5 \%)$ life-threatening conditions. The mortality index was $14.0 \%$ overall, $30.0 \%$ for non-pregnancy-related infections, $2.0 \%$ for obstetric haemorrhage and $13.6 \%$ for hypertension. Of the women with life-threatening conditions, $39.3 \%$ were referred from the primary level of care. Vascular, uterine and coagulation dysfunctions were the most frequent organ dysfunctions in women with life-threatening conditions. The perinatal mortality rate was 26.9/1 000 births overall, 23.1/1 000 for women with non-life-threatening conditions, and 198.0/1 000 for women with life-threatening conditions.

Conclusion. About one in 20 pregnant women in the PAC had a potentially life-threatening condition; $39.3 \%$ of women presented to a primary level facility as an acute emergency and had to be transferred for tertiary care. All healthcare professionals involved in maternity care must have knowledge and skills that equip them to manage obstetric emergencies. Review of the basic antenatal care protocol may be necessary.

S Afr Med J 2015;105(7):578-583. DOI:10.7196/SAMJnew.8038

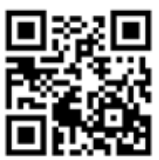

There were 4452 maternal deaths in South Africa (SA) for the period $2011-2013 .^{[1]}$ The institutional maternal mortality ratio (iMMR) in SA decreased from $176.22 / 100000$ live births in the 2008 - 2010 triennium to $154.06 / 100000$ for 2011 - 2013, but further work needs to be done to meet the fifth Millennium Development Goal. In order to reduce maternal mortality, it is important to understand the process of obstetric care, identify weaknesses within the system, and finally implement interventions for improving care. ${ }^{[2]}$

A woman who experiences and survives a severe health condition during pregnancy, during childbirth or after delivery is classified as a maternal near miss. ${ }^{[3]}$ By studying cases of maternal deaths and near misses, important information can be obtained about the processes that take place in healthcare systems responsible for the care of pregnant women. While near-miss cases share many pathological and circumstantial characteristics with maternal deaths, they provide additional information about obstacles that have to be overcome after the onset of an acute complication..$^{[2,4]}$ Although a maternal near-miss case can only be identified retrospectively, it is clinically useful to prospectively identify women with potentially life-threatening conditions. A woman who develops a life-threatening condition will either become a maternal near-miss case or a maternal death.

\section{Objectives}

To determine the spectrum of severe maternal morbidity and mortality in the Pretoria Academic Complex (PAC), SA, and compare the data with previous surveys and the World Health Organization (WHO) Multicountry Survey on Maternal and Newborn Health. ${ }^{[5]}$ The WHO study was used as a comparison because it is the only study to characterise maternal morbidity occurring in a worldwide network of health facilities.

\section{Methods}

This was a descriptive population-based study that took place from 1 August 2013 to 31 July 2014 at nine delivery facilities in central, south-western and eastern Tshwane, Gauteng Province, SA. The following delivery units were included in the study: Steve Biko Academic Hospital (SBAH) (level 3), Kalafong Provincial Tertiary Hospital Hospital (KAH) (level 3), Mamelodi Hospital (level 2), Tshwane District Hospital (TDH) (level 1), Pretoria West Hospital (level 1), Laudium Community Health Centre (CHC) with midwife obstetric unit (MOU), Eersterust MOU, and Stanza Bopape and Dark City clinics (CHCs). SBAH and KAH are tertiary referral hospitals that receive referrals from outside Gauteng, but data were only analysed for women living in the Tshwane region; those living outside were excluded. Cases of abortion and ectopic pregnancy were also 
excluded from the study. Delivery data were recorded on a daily basis at all the health facilities, and daily audit meetings were held at SBAH and $\mathrm{KAH}$ to identify women with life-threatening conditions and organ dysfunction in pregnancy. The following WHO indicators were used to quantify women with severe complications in pregnancy: ${ }^{[2,6]}$

Maternal near miss. A woman who nearly died but survived a complication that occurred during pregnancy or childbirth, or within 42 days of termination of pregnancy. The WHO near-miss criteria are listed in Table 1.

Maternal death. A maternal death is the death of a woman while pregnant or within 42 days of termination of pregnancy, irrespective of the duration and the site of pregnancy, from any cause related to or aggravated by the pregnancy or its management, but not from accidental or incidental causes.

Life-threatening conditions/severe maternal outcome (SMO). This refers to all women who either qualified as having a maternal near miss or who died. It is the sum of maternal near misses and maternal deaths.

Potentially life-threatening condition. The five potentially lifethreatening conditions described by the WHO are severe postpartum haemorrhage, severe pre-eclampsia, eclampsia, sepsis/severe systemic infection, and ruptured uterus. The operational definitions of the five potentially life-threatening conditions are:

- Severe postpartum haemorrhage. Genital bleeding after delivery, with at least one of the following: perceived abnormal bleeding (1 $000 \mathrm{~mL}$ or more) or any bleeding with hypotension or blood transfusion.

- Severe pre-eclampsia. Persistent systolic blood pressure of $\geq 160 \mathrm{mmHg}$ or a diastolic blood pressure of $\geq 110 \mathrm{mmHg}$; proteinuria of $\geq 5 \mathrm{~g}$ in 24 hours; oliguria of $<400 \mathrm{~mL}$ in 24 hours; and HELLP (haemolysis, elevated liver enzymes, low platelets) syndrome or pulmonary oedema. Excludes eclampsia.

- Eclampsia. Generalised fits in a patient without previous history of epilepsy. Includes coma in pre-eclampsia.

- Severe sepsis/systemic infection. Presence of fever (body temperature $>38^{\circ} \mathrm{C}$ ), a confirmed or suspected infection (e.g. chorioamnionitis, septic abortion, endometritis, pneumonia), and at least one of the following: heart rate $>90 \mathrm{bpm}$, respiratory rate $>20 / \mathrm{min}$, leucopenia (white blood cells $<4 \times 10^{\%} / \mathrm{L}$ ), leucocytosis (white blood cells $>12 \times 10^{9} / \mathrm{L}$ )

- Uterine rupture. Rupture of uterus during labour confirmed by laparotomy.

Severe maternal outcome ratio (SMOR). This refers to the number of women with life-threatening conditions per 1000 live births. This indicator gives an estimation of the amount of care that would be needed in an area.

Mortality index (MI). The number of maternal deaths divided by the number of women with life-threatening conditions, expressed as a percentage.

Descriptive statistics in the form of means and standard deviations in the case of continuous data and frequencies and percentages in the case of categorical data were calculated. Ethical approval was obtained from the University of Pretoria Ethics Committee (No. 125/2013).

\section{Results}

There were 26614 deliveries in the PAC (SBAH, KAH, Mamelodi Hospital, TDH, Pretoria West Hospital, Laudium CHC with MOU, Eersterust MOU, Stanza Bopape MOU and Dark City clinics) during the study period. One hundred and thirty-six women developed

\section{Table 1. The WHO near-miss criteria ${ }^{[6}$}

Clinical criteria
Acute cyanosis
Oliguria unresponsive to fluids or diuretics
Jaundice concomitantly with pre-eclampsia
Shock
Cerebrovascular accident
Breathing rate $>40$ - $<6 /$ min
Loss of consciousness, no pulse/heartbeat
Gasping
Coagulation disorders
Total paralysis
Laboratory criteria
Oxygen saturation $<90 \%$ for $>60$ minutes
Creatinine $>300 \mu$ mol/L or $>3.5 \mathrm{mg} / \mathrm{dL}$
Unconscious, presence of glucose and ketoacidosis in urine
PaO $/$ FiO $2<200$ mmHg
Acute thrombocytopenia (platelets $<50 \times 10^{9} / \mathrm{L}$ )
Bilirubin $>100 \mu$ mol/L or $>6.0 \mathrm{mg} / \mathrm{dL}$
Lactate $>5$ mg/dL
pH $<0.1$
$\mathrm{Management}_{2} /$ criteria
Use of continuous vasoactive drug
Puerperal hysterectomy due to infection or haemorrhage
Dialysis for treatment of acute kidney failure
Cardiopulmonary resuscitation

life-threatening conditions, and there were 19 maternal deaths. The SMOR was 5.1/1 000 births and the MI 14.0\%. The caesarean section rate was $25.2 \%$ overall and $61.0 \%$ for women with life-threatening conditions. The HIV infection rate was $19.9 \%$ for the general population, $23.1 \%$ for near misses and $36.8 \%$ for mothers who died. HIV status was unknown in $2.7 \%$ of patients. The spectrum of morbidity from uncomplicated pregnancies to maternal death is illustrated in Fig. 1.

Most of the patients with potentially life-threatening and lifethreatening conditions were treated at the two PAC tertiary level hospitals. Forty-six women (39.3\%) who were classified as near misses and 7 (36.8\%) who died had to be transferred to the tertiary level hospitals after initially presenting at a lower level of care. The most frequent indications for emergency transfer of women with life-threatening conditions to the tertiary hospitals were severe preeclampsia $(15.4 \%, n=21)$, obstetric haemorrhage $(13.2 \%, n=18)$ and organ dysfunction in women with underlying medical disease $(6.6 \%$, $n=9$ ) (Table 2). The MI was $18.6 \%$ for SBAH, $10.2 \%$ for KAH and 12.5\% for Mamelodi Hospital.

Twenty-six women $(22.2 \%)$ who were classified as a near miss had not booked with antenatal care services or had had infrequent visits. Medical practitioners caring for these patients believed that lack of antenatal care may have contributed to the life-threatening event. The distribution of patients with potentially life-threatening 


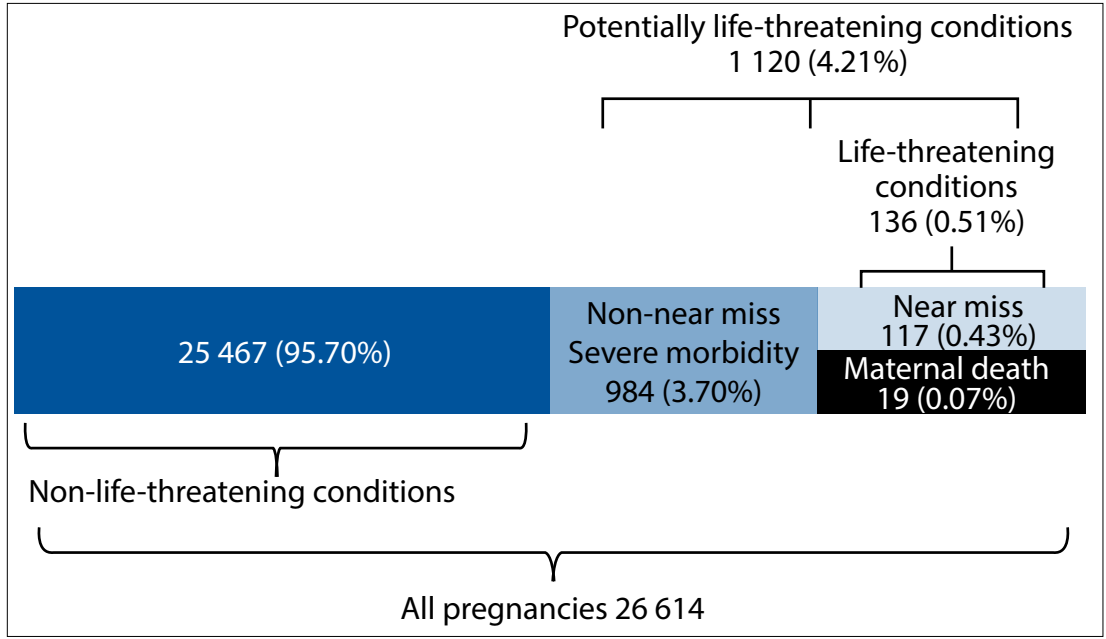

Fig.1. The spectrum of morbidity from uncomplicated pregnancies to maternal death (not drawn to scale).

\section{Table 2. Acute life-threatening conditions necessitating tertiary care $(N=136)$}

\begin{tabular}{lll}
\hline Condition & $\begin{array}{l}\text { Patients referred to tertiary } \\
\text { centre from lower levels of care } \\
\boldsymbol{n}(\%)\end{array}$ & $\begin{array}{l}\text { Patients already } \\
\text { in tertiary care } \\
\boldsymbol{n}(\%)\end{array}$ \\
\hline Obstetric haemorrhage & $18(13.2)$ & $26(19.1)$ \\
Pre-eclampsia & $21(15.4)$ & $22(16.2)$ \\
Sepsis & $3(2.2)$ & $11(8.1)$ \\
Medical/surgical disorders & $9(6.6)$ & $9(6.6)$ \\
Non-pregnancy-related infections & $3(2.2)$ & $8(5.9)$ \\
Anaesthetic disorders & - & $4(2.9)$ \\
Other & - & $2(1.5)$ \\
Total & $54(39.7)$ & $82(60.3)$
\end{tabular}

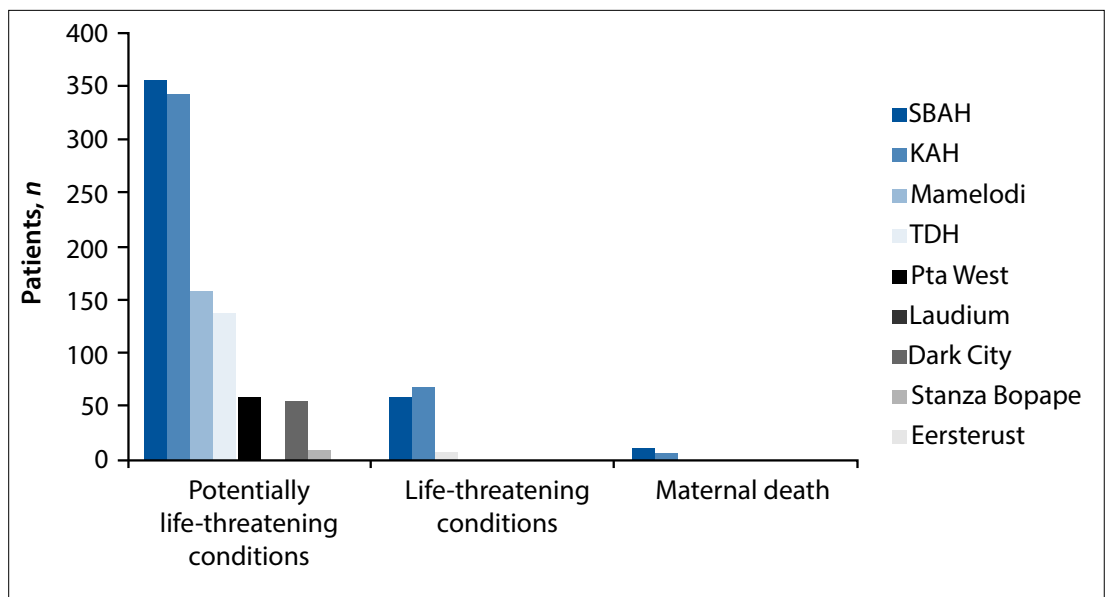

Fig. 2. Distribution of potentially life-threatening conditions, near misses and maternal deaths in relation to the different levels of care.

conditions in relation to the different levels of care is shown in Fig. 2.

The frequency of potentially life-threatening disorders is shown in Table 3. (Antepartum haemorrhage and non-pregnancy-related infections that are not part of the WHO definition of potentially life-threatening conditions have been included.)
The MI for non-pregnancy-related infections was $30.0 \%$, for obstetric haemorrhage $2.0 \%$, for hypertension $13.6 \%$ and for medical and surgical disorders $19.0 \%$ (Table 4 ).

The near-miss markers and distribution of organ dysfunction are shown in Tables 5 and 6 . The average age of the women who were classified as a near miss was
30.3 years (minimum 16, maximum 43 ); 34 (29.1\%) were primigravidas and $83(70.9 \%)$ were multigravidas. There were 6 maternal deaths related to HIV and AIDS; 4 patients had respiratory failure secondary to $\mathrm{TB}$ pneumonia, 1 had bacterial meningitis and 1 died after presenting with multiorgan failure and milliary tuberculosis. Six women died of complications related to pre-eclampsia: 1 patient had a liver rupture, 2 had intracranial bleeds, 2 had respiratory failure due to pulmonary oedema and 1 had a cardiac arrest. The patient who died as a result of postpartum haemorrhage had a placenta praevia and had had two previous caesarean sections. Although an antenatal ultrasound scan had confirmed the location of the placenta, the diagnosis of placenta accreta was missed. Surgeons encountered a major bleed at caesarean section, and despite two re-look laparotomies the bleeding could not be controlled. Four patients died due to underlying medical disease, 1 each due to breast cancer, acute-on-chronic pancreatitis and an anaesthetic complication in a diabetic patient, while the 4 th patient had a prosthetic heart valve.

There were no maternal deaths at the level 1 hospitals or CHCs and only one death at a level 2 hospital. This was a patient with advanced-stage breast cancer who was unable to obtain transport to a tertiary-level facility. The MIs for the two tertiary hospitals were $18.6 \%(\mathrm{SBAH})$ and $10.15 \%(\mathrm{KAH})$, and that for the level 2 hospital was $12.5 \%$.

Table 7 compares the indices of severe acute maternal morbidity at the PAC for the periods $1997-1998,2002-2004$ and 2013 - 2014. Although the SMOR for the general population has remained the same since 1997 - 1998, both the iMMR and the MI have decreased. These findings are consistent for postpartum haemorrhage and hypertension. The SMOR for puerperal sepsis has remained constant despite the HIV epidemic with a decrease in MI. The SMOR and MI for medical and surgical conditions remain unchanged.

Fig. 3 illustrates the perinatal mortality related to maternal morbidity. The women with severe maternal morbidity and mortality had a much high perinatal mortality rate (PNMR); however, for every woman with a complicated pregnancy, almost five women had no life-threatening condition. This explains the relatively small difference between the total PNMR and the PNMR of the non-life-threatening conditions.

The primary obstetric causes of perinatal death were unexplained intrauterine death (30.3\%), spontaneous preterm labour (25.5\%), antepartum haemorrhage 
(12.3\%), intrapartum asphyxia (9.3\%) hypertensive disorders (7.4\%), fetal abnormality (6.9\%) and maternal disease (3.7\%).

\section{Discussion}

To our knowledge, this is the first study in SA assessing the spectrum of morbidity

\section{Table 3. Frequency of potentially life-threatening disorders}

\begin{tabular}{|c|c|c|c|}
\hline & $\begin{array}{l}\text { All women } \\
(N=26614) \\
n(\%)\end{array}$ & $\begin{array}{l}\text { Women with SMO } \\
(N=136) \\
n(\%)\end{array}$ & $\begin{array}{l}\text { HIV infection in } \\
\text { women with SMO } \\
n(\%)\end{array}$ \\
\hline Severe haemorrhage & $660(2.5)$ & $51(37.5)$ & $7(13.7)$ \\
\hline Antepartum haemorrhage & $301(1.1)$ & $17(12.5)$ & $1(5.9)$ \\
\hline Postpartum haemorrhage & $336(1.3)$ & $31(22.7)$ & $4(12.9)$ \\
\hline Ruptured uterus & $23(0.1)$ & $3(2.2)$ & $2(66.7)$ \\
\hline Severe hypertensive disorders & $682(2.6)$ & $44(32.4)$ & $4(9.1)$ \\
\hline Pre-eclampsia & 457 (1.7) & $40(29.4)$ & $4(10.0)$ \\
\hline Eclampsia & $225(0.8)$ & $4(2.9)$ & - \\
\hline \multicolumn{4}{|l|}{ Other complications } \\
\hline Puerperal sepsis & $35(0.1)$ & $14(10.3)$ & $2(14.3)$ \\
\hline Non-pregnancy-related infections & & $20(14.7)$ & $20(100.0)$ \\
\hline
\end{tabular}

\section{Table 4. MIs for different disease conditions}

\begin{tabular}{llll}
\hline Underlying condition & $\begin{array}{l}\text { Maternal } \\
\text { near miss, } \boldsymbol{n}\end{array}$ & $\begin{array}{l}\text { Maternal } \\
\text { death, } \boldsymbol{n}\end{array}$ & MI, \% \\
\hline Obstetric haemorrhage & 50 & 1 & 2.0 \\
$\quad$ Antepartum haemorrhage & 17 & 0 & 0 \\
Ruptured uterus & 3 & 0 & 0 \\
Postpartum haemorrhage & 30 & 1 & 3.2 \\
Hypertension & 38 & 6 & 13.6 \\
Chronic & 1 & 0 & 0 \\
Pre-eclampsia & 35 & 4 & 10.0 \\
Eclampsia & 2 & 2 & 50.0 \\
Puerperal sepsis & 14 & 0 & 0 \\
Non-pregnancy-related infections & 14 & 6 & 30.0 \\
Medical/surgical disorders & 17 & 4 & 19.0
\end{tabular}

Table 5. Markers for classification of a maternal near miss $(N=117)$

\begin{tabular}{ll}
\hline Near-miss marker & $\boldsymbol{n}(\%)$ \\
\hline Cerebrovascular accident & $2(1.7)$ \\
Total paralysis & $1(0.9)$ \\
Oxygen saturation $<90 \%$ for $>60$ minutes & $6(5.1)$ \\
Acute thrombocytopenia (platelets $\left.<50 \times 10^{9} / \mathrm{L}\right)$ & $26(2.2)$ \\
Creatinine $>300 \mu \mathrm{mol} / \mathrm{L}$ or $>3.5 \mathrm{mg} / \mathrm{dL}$ & $4(3.4)$ \\
Bilirubin $>100 \mu \mathrm{mol} / \mathrm{L}$ or $>6.0 \mathrm{mg} / \mathrm{dL}$ & $1(0.9)$ \\
Ketoacids in urine & $4(3.4)$ \\
Use of continuous vasoactive drug & $3(2.6)$ \\
Dialysis for acute renal failure & $2(1.7)$ \\
Hysterectomy following infection or haemorrhage & $35(29.9)$ (infection 14, haemorrhage 21$)$ \\
Cardiopulmonary resuscitation & $3(2.6)$ \\
Transfusion of $>5$ units red cells & $31(26.5)$ \\
Intubation and ventilation for $>60$ minutes & $18(15.4)$
\end{tabular}

for a specific region. There were 26614 deliveries over a 12-month period (2013 2014). This represents almost a doubling of deliveries since 1997 - 1998, when the total number of births for the biennium was 27025 , and a $35 \%$ increase since 2002 - 2004 (51 469 births). ${ }^{[7]}$ Just over $4 \%$ of women developed a potentially lifethreatening condition and $0.5 \%$ developed a life-threatening condition. This is lower than the WHO Multicountry Survey on Maternal and Newborn Health, which reported an incidence of $7 \%$ for potentially life-threatening conditions and $1 \%$ for lifethreatening conditions. ${ }^{[5]}$ However, the difference between the two studies was that ours was population based while the WHO study was hospital based.

About $40 \%$ of women with acute lifethreatening conditions did not present directly to the two tertiary level hospitals during the acute stage of disease. These patients were booked at a level 1 or 2 facility and then developed an acute condition requiring urgent transfer. Similarly, the England Collaborative Group reported that a significant proportion of serious complications occur in women with no recognisable risk factors. ${ }^{[8]}$ Severe preeclampsia, obstetric haemorrhage and organ dysfunction due to an underlying medical condition were the most important reasons for emergency transfer. This indicates the need to have all healthcare professionals involved in care of pregnant women trained in the initial stabilisation and management of obstetric and neonatal emergencies. The antenatal care protocol used in our complex is based on the WHO recommendation of four antenatal visits for low-risk patients. ${ }^{[9]}$ The low frequency of visits possibly means

Table 6. Organ system dysfunction in women with life-threatening conditions $(N=136)^{*}$

\begin{tabular}{lc}
\hline Dysfunction & $\boldsymbol{n}(\%)$ \\
\hline Vascular dysfunction & $54(39.7)$ \\
(hypovolaemia) & $35(25.7)$ \\
Uterine dysfunction & $27(19.9)$ \\
Coagulation dysfunction & $24(17.7)$ \\
Respiratory dysfunction & $9(6.6)$ \\
Cardiovascular dysfunction & $8(5.9)$ \\
Immunological dysfunction & $8(5.9)$ \\
Renal dysfunction & $7(5.2)$ \\
Cerebral dysfunction & $5(3.7)$ \\
Hepatic dysfunction & $5(3.7)$ \\
Metabolic dysfunction & *Some women had more than one organ dysfunction.
\end{tabular}


Table 7. Comparison of the indices of severe acute morbidity rates at the PAC for the periods 1997 - 1998, $2002-2004$ and 2013 - 2014

\begin{tabular}{|c|c|c|c|c|c|c|c|c|c|}
\hline & \multicolumn{3}{|c|}{$1997-1998$} & \multicolumn{3}{|c|}{$2002-2004$} & \multicolumn{3}{|c|}{$2013-2014$} \\
\hline & SMOR & iMMR & MI & SMOR & iMMR & MI & SMOR & iMMR & MI \\
\hline Antepartum haemorrhage & 1.0 & 0 & 0 & 0.9 & 1.9 & 2.1 & 0.6 & 0 & 0 \\
\hline Postpartum haemorrhage & 1.4 & 7.4 & 5.3 & 2.1 & 15.5 & 7.5 & 1.2 & 3.8 & 3.2 \\
\hline Hypertension & 1.5 & 33.3 & 22.5 & 1.57 & 19.4 & 12.3 & 1.6 & 22.5 & 13.6 \\
\hline Puerperal sepsis & 0.4 & 7.4 & 20.0 & 0.5 & 5.8 & 10.7 & 0.5 & 0 & 0 \\
\hline Non-pregnancy-related infections & 0.3 & 22.2 & 66.7 & 0.4 & 19.4 & 47.6 & 0.8 & 22.5 & 30.0 \\
\hline Medical and surgical disorders & 0.8 & 11.1 & 14.3 & 0.8 & 11.7 & 14.3 & 0.8 & 15.0 & 19.0 \\
\hline Total (excluding early pregnancy losses) & 5.8 & 96.2 & 16.6 & 7.0 & 85.5 & 12.2 & 5.1 & 71.4 & 14.0 \\
\hline
\end{tabular}

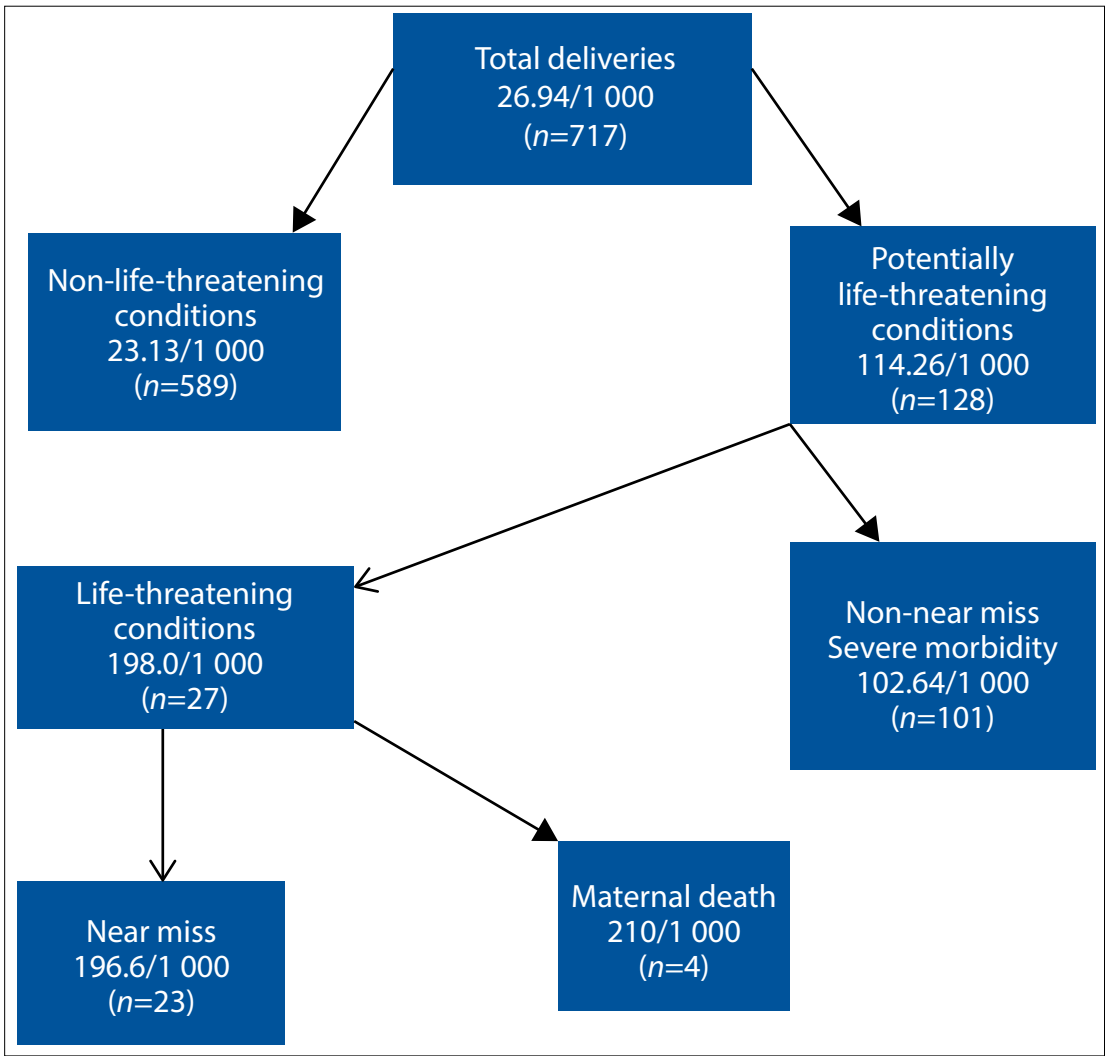

Fig. 3. Perinatal mortality rate (for babies $>500 \mathrm{~g}$ ).

that cases of pre-eclampsia in the early stages of the disease process were missed, leading to patients presenting at a later stage with acute complications requiring tertiary care. Early detection of pre-eclampsia may require revision of our current antenatal care protocol and is supported by the recent Cochrane review on patterns of routine antenatal care for low-risk pregnancy. ${ }^{[10]}$

There has been a decrease in the iMMR and MI at the PAC since 1997. This has been associated with decreases in MI for postpartum haemorrhage, hypertension, puerperal sepsis and non-pregnancy-related infections. The MI for non-pregnancyrelated infections in the PAC was $66.7 \%$ in $1997-1999,75 \%$ in $2000,47.6 \%$ in
$2002-2004$ and $30 \%$ in $2013-2014 .^{[8,11]}$ The decrease reflects the implementation of the antiretroviral programme by the National Department of Health and better handling of respiratory complications. Of significance is the low MI for postpartum haemorrhage (3.2\%), which is less than half of the rate $(7.5 \%)$ reported in $2002-2004$ and significantly lower than the rate $(5.3 \%)$ reported for 1997 - 1998. ${ }^{[11,12]}$ The decrease in MI for severe postpartum haemorrhage and puerperal sepsis is probably a result of the introduction of strict protocols. The frequency of postpartum haemorrhage in women with life-threatening conditions $(22.7 \%)$ was similar to that in the WHO study (26.7\%). ${ }^{[6]}$ However, the rates of pre- eclampsia (29.4\%) and non-pregnancyrelated infections $(14.7 \%)$ were greater in our study (WHO $16.3 \%$ and $1.6 \%$, respectively). The rate of pre-eclampsia in women with life-threatening conditions was consistent with reports from Nigeria (32.5\%) and Mozambique (32.9\%). ${ }^{[13,14]}$

Vascular (hypovolaemia), uterine (hysterectomy) and coagulation (low platelets) dysfunction were the most frequent organ system dysfunctions in women with lifethreatening conditions (Table 6). Many women had multiple complications. The disease profile in our complex has changed since the year 2000, when vascular, cardiac, immunological and coagulation dysfunction were the most important organ systems causing obstetric morbidity. ${ }^{[15]}$ Obstetric haemorrhage was the potentially lifethreatening condition most frequently encountered in our complex (37.5\%), and vascular dysfunction as a result of hypovolaemia was the most common organ system dysfunction seen. The low MI for postpartum haemorrhage suggests that although postpartum haemorrhage is an important problem, the condition is well managed by our clinicians.

Of the five potentially life-threatening conditions, hypertensive disorders contributed to $7.4 \%$ of perinatal deaths. Ninety-three per cent of perinatal deaths were not related to antepartum and intrapartum maternal lifethreatening conditions, and if postpartum maternal life-threatening conditions are included, $80 \%$ of the women with perinatal deaths did not have severe morbidity. These findings are consistent with those of Allanson et al. ${ }^{[16]}$ describing perinatal mortality in Mpumalanga Province, SA, and Vogel et $a l^{[17]}$ in the WHO Multicountry Survey, who found that a significant proportion of women have no recognisable obstetric or medical condition at the time of perinatal death. Allanson et al. ${ }^{[16]}$ found a rate of maternal complications in macerated stillbirths, fresh stillbirths and early neonatal deaths of $50.4 \%$, 
$50.7 \%$ and $25.8 \%$, respectively. The WHO Multicountry Survey found a maternal complication rate of $22.9 \%, 27.7 \%$ and $21.2 \%$ in late macerated stillbirths, late fresh stillbirths and early neonatal deaths, respectively. Current early antenatal identification of both severe maternal morbidity and perinatal mortality is inadequate.

\section{Study strengths and limitations}

The strength of this study is the robust method of data collection. The new national birth register records maternal complications, facilitating the collection of data. The PAC has been collecting and reviewing data on life-threatening conditions for more than 15 years, and all doctors are familiar with the WHO near-miss criteria. Women who were classified as a near miss were interviewed about barriers encountered in accessing healthcare. This information will be presented in a separate article.

A limitation of this study is the exclusion of cases of early pregnancy loss (abortions and ectopic pregnancies). Some cases of sepsis may have been missed if patients presented late in the postpartum period. Furthermore, maternal infections such as pneumonia, tuberculosis and meningitis were not on the list of potentially life-threatening conditions, so the SMOR could not be calculated for these disease conditions. The list of potentially life-threatening conditions should be expanded to include medical conditions and non-pregnancyrelated infections. This is supported by Lumbiganon et al. ${ }^{[18]}$ who demonstrated that indirect causes of maternal deaths are increasingly important in developing countries, with indirect causes being responsible for about one-fifth of severe maternal outcomes.

\section{Recommendations}

- The WHO has identified five potentially life-threatening conditions: severe postpartum haemorrhage, severe pre-eclampsia, eclampsia, sepsis/severe infection and ruptured uterus. ${ }^{[2]}$ Our study has shown that conditions such as abruptio placentae, nonpregnancy-related infections and medical and surgical disorders are also important causes of obstetric morbidity, and the WHO should therefore consider expanding its categories of potentially life-threatening conditions.

- Forty per cent of patients with life-threatening conditions presented to a level 1 or 2 facility before being transferred for tertiary care. Cases of postpartum haemorrhage and severe pre-eclampsia could not be predicted antenatally. In addition, no recognisable obstetric condition was present in the majority of pregnancies that ended in a perinatal death. Health workers in level 1 and 2 centres must therefore be able to recognise, stabilise and transfer pregnant women and neonates presenting with an acute obstetric emergency.

- Strategies to prevent and screen for pre-eclampsia and improvement of emergency transport for women are essential in order to reduce obstetric morbidity and mortality.

- Review of the reduced visits protocol put forward by the WHO should be considered, as increasing the frequency of antenatal visits for low-risk women may increase detection of pre-eclampsia at an earlier stage of the disease process. ${ }^{[8]}$ However, this would require a considerable increase in resources.

\section{Conclusion}

In this study we were able to identify the proportion of pregnancyrelated morbidity in our population and compare it with other studies. The MI and prevalence of potentially life-threatening conditions were similar to those in the WHO Multicountry Survey. Although there has been a decrease in the MI for non-pregnancy-related infection, further interventions need to be implemented to reduce morbidity and mortality associated with HIV disease and tuberculosis. A significant proportion of women who developed severe maternal conditions were not identified during the antenatal period, indicating the need to ensure that all levels of care can manage the initial steps in obstetric and neonatal emergencies and that an efficient emergency transport system is available.

\section{References}

1. Pattinson RC, ed. Saving Mothers 2011-2013: Sixth Report on Confidential Enquiries into Maternal Deaths in South Africa. Pretoria: Department of Health, 2014.

2. World Health Organization. Evaluating the Quality of Care for Severe Pregnancy Complications. The WHO Near-miss Approach for Maternal Health. Geneva: WHO Press, 2011.

3. Pattinson RC, Hall MH. Near Misses: A useful adjunct to maternal death enquiries. Br Med Bull 2003;67(1):231-243. [http://dx.doi.org/10.1093/bmb/ldg007]

4. Chhabra P. Maternal near miss: An indicator for maternal health and maternal care. Indian J Community Med 2014;39(3):132-137. [http://dx.doi.org/10.4103/0970-0218.137145]

5. Souza JP, Gulmezoglu AM, Vogel J, et al. Moving beyond essential interventions for reduction of 5. Souza JP, Gulmezoglu AM, Vogel J, et al. Moving beyond essential interventions for reduction of
maternal morbidity (the WHO Multicountry Survey on Maternal and Newborn Health): A crossmaternal morbidity (the WHO Multicountry Survey on Maternal and Newborn Health): A cross-
sectional study. Lancet 2013;381(9879):1747-1755. [http://dx.doi.org/10.1016/S0140-6736(13)60686-8] 6. Say L, Souza JP, Pattinson RC. Maternal near-miss - towards a standard tool for monitoring quality of maternal care. Best Pract Res Clin Obstet Gynaecol 2009;23(3):287-296. [http://dx.doi.org/10.1016/j. bpobgyn.2009.01.007]

7. Pattinson RC, Macdonald AP, Backer F, Kleynhans M. Effect of audit on critically ill pregnant women. Clinical Governance: An International Journal 2006;11(4):278-288. [http://dx.doi. org/10.1108/14777270610708814.

8. Birthplace in England Collaborative Group. Perinatal and maternal outcomes by planned place of birth for healthy women with low risk pregnancies: The Birthplace in England national prospective cohort study. BMJ 2011;343:d7400. [http://dx.doi.org/10.1136/bmj.d7400]

9. Villar J, Bergsjo P. WHO Antenatal Care Randomised Trial: Manual for the Implementation of the New Model. Geneva: WHO, 2003

10. Dowswell T, Carroli G, Duley L, et al. Alternative versus standard packages of antenatal care for lowrisk pregnancy (Review). Cochrane Database Syst Rev 2010, Issue 10. Art. No.: CD000934. [http:// dx.doi.org/10.1002/14651858.CD000934.pub2]

11. Vandecruys HIB, Pattinson RC, Macdonald AP, Mantel GD. Severe acute maternal morbidity and mortality in the Pretoria Academic Complex: Changing patterns over 4 years. Eur J Obstet Gynecol Reprod Biol 2002;102(1):6-10. [http://dx.doi.org/10.1016/S0301-2115(01)00558-9]

12. Lombaard H, Pattinson RC. Common errors and remedies in managing postpartum haemorrhage. Best Pract Res Clin Obstet Gynaecol 2009;23(3):317-326. [http://dx.doi.org/10.1016/j. bpobgyn.2009.01.006

13. Daru PH, MU J, Achara P, et al. Near miss maternal mortality in Jos University Teaching Hospital, Jos, Plateau State Nigeria. Ibom Medical Journal 2008;3(1):18-21.

14. David E, Machungo F, Zanconato G et al. Maternal near miss and maternal deaths in Mozambique: A cross sectional, region-wide study of 635 consecutive cases assisted in health facilities of Maputo Province. BMC Pregnancy Childbirth 2014;14:401. [http://dx.doi.org/10.1186/s12884-014-0401-3]

15. Mantel GD, Buchmann E, Rees H, Pattinson RC. Severe acute maternal morbidity: A pilot study of a definition for a near-miss. BJOG 1998;105(9):985-990. [http://dx.doi.org/10.1111/j. 1471-0528]

definition for a near-miss. BJOG 1998;105(9):985-990. [http://dx.doi.org/10.1111/j. 1471-0528]
16. Allanson ER, Muller M, Pattinson RC. Causes of perinatal mortality and associated maternal Allanson ER, Muller M, Pattinson RC. Causes of perinatal mortality and associated maternal
complications in a South African province: Challenges in predicting poor outcomes. BMC Pregnancy complications in a South African province: Challenges in predicting po

17. Vogel JP, Souza JP, Mori R, et al. Maternal complications and perinatal mortality: Findings of the World health Organisation Multicountry Survey on Maternal and Newborn Health. BJOG 2014;121(Suppl. 1):76-88. [http://dx.doi.org/10.1111/1471-0528.12633]

18. Lumbiganon $P$, Laopaiboon $M$, Intarut $\mathrm{N}$, et al. Indirect causes of severe adverse maternal outcomes: A secondary analysis of the WHO Multicountry Survey on Maternal and Newborn Health. BJOG 2014;121(Suppl. 1):32-39. [http://dx.doi.org/10.1111/1471-0528.12647]

Accepted 27 March 2015. 